\title{
Ciclo vigília/sono e o transtorno de déficit de atenção/hiperatividade
}

\author{
Sleep/wake cycle and the attention deficit/hyperactivity disorder
}

Tâmile Stella Anacleto ${ }^{1}$, Fernando Mazzilli Louzada², Érico Felden Pereira ${ }^{3}$

RESUMO

Objetivo: Analisar as relações entre ciclo vigília/sono e transtorno de déficit de atenção/hiperatividade.

Fontes de dados: Os artigos foram selecionados nas bases de dados SciELO e PubMed, utilizando-se “sono”, "transtorno de déficit de atenção/hiperatividade", "atividade motora” e "crianças" como palavras-chave para a busca.

Síntese dos dados: Embora os resultados de diferentes estudos sejam inconclusivos e, por vezes, contraditórios, não sendo possível estabelecer relações claras entre sono e transtorno do déficit de atenção/hiperatividade, observase que o sono das crianças diagnosticadas difere do sono de crianças que não possuem o transtorno, sugerindo que alterações de sono possam se relacionar ao transtorno do déficit de atenção/hiperatividade.

Conclusões: A falta de marcadores biológicos e de conhecimento a respeito da sua etiologia gera dificuldades na determinação da real prevalência do transtorno do déficit de atenção/hiperatividade, além de limitar sua compreensão e a busca por novas formas de tratamento e prevenção. Embora dificuldades de sono sejam frequentemente relatadas na prática clínica e já tenham sido utilizadas como um dos critérios diagnósticos para o transtorno, pouco se sabe sobre a possível participação da privação de sono na etiologia do transtorno do déficit de atenção/hiperatividade.

Palavras-chave: transtorno de déficit de atenção/hiperatividade; sono; atividade motora; criança.

\section{ABSTRACT}

Objective: To analyze the relationship between the wake/ sleep cycle and the attention deficit/hyperactivity disorder.

Data Source:The articles were found in the SciELO and PubMed databases, using "sleep", "attention deficit/ hyperactivity disorder", "motor activity" and "children" as search key-words.

Data Synthesis: The results of individual studies are inconclusive and frequently contradictory, not allowing the establishment of a clear relationship between sleep and the attention deficit/hyperactivity disorder. However, sleep patterns of children diagnosed with this disorder are different than those of healthy children. Thus, the possible role of sleep alterations in attention deficit/hyperactivity disorder etiology cannot be ruled out.

Conclusions: The lack of biological markers and knowledge about the etiology of the attention deficit/hyperactivity disorder results in some difficulties in determining its real prevalence. Moreover, this restricts our understanding of the attention deficit/hyperactivity disorder and the search for new forms of treatment and prevention. Although sleeping difficulties have been frequently mentioned in clinical practice studies and are already being used as one of the diagnostic criteria for the attention deficit/hyperactivity disorder, little is known about the possible participation of sleep deprivation in the etiology of the disorder.

Key-words: attention deficit/hyperactivity disorder; sleep; motor activity; child.
Instituição: Laboratório de Cronobiologia do Departamento de Fisiologia da Universidade Federal do Paraná (UFPR), Curitiba, PR, Brasil

${ }^{1}$ Mestre em Biologia Celular e Molecular pela UFPR, Curitiba, PR, Brasil

${ }^{2}$ Pós-Doutor pela Harvard Medical School; Pesquisador do Laboratório de Cronobiologia do Departamento de Fisiologia da UFPR, Curitiba, PR, Brasil

${ }^{3}$ Mestre em Educação Física pela Universidade Federal de Santa Catarina (UFSC), Florianópolis, SC, Brasil
Endereço para correspondência:

Tâmile Stella Anacleto

Rua Coronel Francisco Heráclito dos Santos, 210 - Jardim das Américas CEP 81531-980 - Curitiba/PR

E-mail: tami_anac@hotmail.com

Conflito de interesse: nada a declarar

Recebido em: 28/2/2010

Aprovado em: 20/9/2010 


\section{Introdução}

O transtorno de déficit de atenção/hiperatividade (TDAH) foi inicialmente descrito em 1902, quando George Still relatou o comportamento impulsivo e hiperativo de 43 crianças. Desde então, sofreu diversas mudanças de nomenclatura até 1994 , quando chegou à denominação atual ${ }^{(1,2)}$. Hoje, o TDAH é considerado o distúrbio neurocomportamental mais comum e socialmente importante em crianças e adolescentes na idade escolar ${ }^{(3-7)}$.

Segundo a quarta edição do Manual Diagnóstico e Estatístico de Transtornos Mentais (DSM-IV) da Associação Americana de Psiquiatria ${ }^{(8)}$, a característica essencial do TDAH é um padrão persistente de desatenção e/ou hiperatividade, mais frequente e grave do que aquele tipicamente observado em indivíduos em nível equivalente de desenvolvimento. Os sintomas do TDAH incluem também déficits em outras funções, tais como organização e memória de trabalho, além de impulsividade comportamental aumentada e agitação motora $^{(9)}$. Embora a maioria dos indivíduos apresente sintomas tanto de desatenção quanto de hiperatividade-impulsividade, existem indivíduos nos quais há predomínio de um ou outro padrão. Com a proximidade da adolescência, é comum, no entanto, que os sintomas de hiperatividade diminuam, acentuando-se a desatenção e a impulsividade ${ }^{(10)}$.

Não existe um marcador biológico, genético, neuroquímico ou de neuroimagem que permita a confirmação da presença do transtorno. Assim, o diagnóstico, feito geralmente na faixa etária escolar, é clínico, determinado a partir dos sintomas de desatenção, hiperatividade e impulsividade estabelecidos pelo DSM-IV. Tais sintomas manifestam-se em dois locais distintos, geralmente na escola e em casa, por pelo menos seis meses ${ }^{(3,2)}$.

A definição diagnóstica é um dos motivos pelos quais os estudos divergem em relação à prevalência do transtorno. Cormier relata que esses números podem variar de 2 a $16 \%$, em função dos critérios e instrumentos utilizados no diagnóstico ${ }^{(11)}$. Há pouca investigação da prevalência do transtorno no Brasil. Fontana et al, a partir de dados de 461 alunos com idade entre seis e 12 anos, de quatro escolas públicas brasileiras, identificaram prevalência de $13 \%{ }^{(12)}$.

Com relação à etiologia do transtorno, ainda não existe consenso. Entretanto, dada sua complexidade e amplitude de sinais e sintomas, pode-se imaginar que sejam múltiplas as causas e fatores de risco implicados. Segundo Singh, existiriam três visões a respeito da origem do TDAH. Uma delas considera-o como um transtorno primariamente causado por uma combinação de fatores biológicos associados, que levariam a um desequilíbrio neuroquímico capaz de afetar as funções motoras e cognitivas. Outra visão considera que o transtorno seja causado por uma combinação de fatores sociais e biológicos e, por fim, uma terceira considera que o transtorno seja resultado de causas ambientais primárias, tais como o tabagismo durante a gestação, exposição ao chumbo e ingestão de aditivos presentes nos alimentos ${ }^{(13)}$.

Entre os fatores biológicos, estudos sugerem que alterações nos genes transportador (DAT) e receptor (DRD4) da dopamina, assim como disfunções noradrenérgicas no córtex pré-frontal, estejam relacionadas à suscetibilidade ao $\mathrm{TDAH}^{(2)}$. De fato, o metilfenidato, fármaco amplamente utilizado no tratamento do transtorno, atua bloqueando a reabsorção da dopamina e da noradrenalina no terminal présináptico, mantendo níveis extracelulares elevados ${ }^{(3,14)}$. Há relatos de que a administração de metilfenidato leva à redução dos sintomas, produz melhora no desempenho de tarefas que requerem vigilância, assim como diminui a sensibilidade à fadiga ${ }^{(15)}$. Entretanto, a presença de pacientes portadores de TDAH que não apresentam tais alterações gênicas, bem como a existência de diversos fatores capazes de gerar desequilíbrio funcional do córtex pré-frontal, endossa a suspeita de etiologia multifatorial para o transtorno ${ }^{(2)}$.

O diagnóstico de diversas comorbidades associadas é também característico do transtorno. Segundo Tomás Vila et al, $50 \%$ das crianças com TDAH apresentam comorbidades associadas, podendo-se destacar o transtorno oposicional desafiante e o transtorno de conduta, entre outras alterações psíquicas. Entretanto, segundo tais autores, os distúrbios de sono costumam estar entre as comorbidades mais comumente associadas ao transtorno de déficit de atenção e hiperatividade ${ }^{(16)}$.

Dada a dificuldade de diagnóstico e a necessidade de maiores investigações sobre a etiologia do transtorno e a frequente relação, observada na prática clínica, entre problemas de sono em crianças com TDAH, buscou-se analisar os artigos publicados com pesquisas que investigaram as possíveis relações entre características do sono e TDAH. Para tanto, foi realizada uma busca exploratória nas bases de dados SciELO e PubMed, tendo como palavras-chave para a busca termos como "sono", "transtorno de déficit de atenção/ hiperatividade" "atividade motora" e "crianças".

\section{TDAH e distúrbios do sono}

Embora a função do sono ainda não seja totalmente conhecida ${ }^{(17)}$, estudos realizados nas últimas décadas mostraram que o sono é um estado de consciência fundamental 
para a regulação do metabolismo energético, consolidação da memória, termorregulação e plasticidade neural ${ }^{(18-20)}$. Alterações metabólicas ${ }^{(21)}$, déficit de memória ${ }^{(22)}$ e redução do desempenho cognitivo ${ }^{(23)}$ são algumas das consequências da sua privação.

Além do conhecimento sobre as alterações cognitivas e comportamentais decorrentes da privação de sono, as quais têm impacto negativo no desempenho acadêmico de crianças e adolescentes ${ }^{(24-26)}$, a redução das horas de sono também tem sido identificada como um dos fatores associados ao transtorno do déficit de atenção e hiperatividade (TDAH) em crianças e adolescentes ${ }^{(27-29)}$.

Segundo Biederman e Spencer ${ }^{(30)}$, alterações em vias neurotransmissoras noradrenérgicas e dopaminérgicas e descritas em portadores de TDAH, assim como mudanças metabólicas do córtex pré-frontal ${ }^{(31)}$, são também encontradas em pacientes com distúrbios de sono. De fato, dificuldades para início e manutenção do sono são frequentemente relatadas em crianças e adolescentes com TDAH; o sono agitado é um dos critérios listados para o diagnóstico do transtorno na terceira edição do Manual Estatístico e Diagnóstico de Transtornos Mentais. Entretanto, esse critério foi eliminado das edições subsequentes do $\operatorname{DSM}^{(6)}$.

Dessa forma, diversos estudos compararam o sono de crianças com TDAH e sem o transtorno, por meio de medidas objetivas (polissonografia, gravação de vídeo e actimetria) e subjetivas (preenchimento de questionários pelos pais sobre os hábitos de sono da criança). Alguns desses estudos apontaram que crianças com TDAH têm latência de sono aumentada, porcentagem de sono REM diminuída ${ }^{(32)}$ e aumento da atividade motora noturna ${ }^{(33)}$.

Por meio do uso de gravação de vídeo e polissonografia, Konofal et al avaliaram os padrões de atividade motora durante o sono de 30 crianças com TDAH e 19 controles saudáveis. A média de duração dos movimentos executados durante o sono foi cerca de duas vezes maior nas crianças com TDAH do que nas crianças saudáveis, para todos os tipos de movimentos. Outro importante achado do mesmo estudo foi a correlação positiva entre a pontuação da escala Conner, por meio da qual pais e professores avaliam a presença de sintomas do TDAH e a duração dos movimentos durante o sono. Crianças mais agitadas durante o dia apresentam elevados padrões de atividade noturna. Dessa forma, o TDAH pode ser um transtorno que afeta não somente o comportamento diário, mas também mecanismos relacionados ao sono ${ }^{(33)}$.

Thunström, em um estudo longitudinal, comparou um grupo de 27 crianças com idade entre seis e 12 meses, que preenchiam critérios específicos para problemas graves e crônicos do sono, com um grupo controle de 27 crianças de mesma idade e sexo. Aos cinco anos e meio, uma em cada quatro crianças daquelas pertencentes ao grupo que apresentava problemas de sono mostrou critérios diagnósticos para TDAH, enquanto nenhuma das pertencentes ao grupo controle recebeu tal diagnóstico, sugerindo que a presença de problemas de sono durante a infância possa estar relacionada ao desenvolvimento do $\mathrm{TDAH}^{(27)}$.

Touchette et al, em estudo longitudinal que acompanhou crianças entre dois anos e meio e seis anos de idade, observaram que a menor duração de sono antes dos três anos e meio estava associada à hiperatividade e pior desempenho cognitivo entre cinco e seis anos ${ }^{(28)}$.

Entretanto, Cortese et al chamam a atenção para o fato de que, enquanto alguns autores encontraram diferenças significativas entre os padrões de sono de crianças portadoras e não portadoras do TDAH, outros não identificaram tais diferenças $^{(34,35)}$. Dessa forma, seria inapropriado utilizar esses resultados para elaborar conclusões globais a respeito do assunto. Os autores explicam que tais diferenças são causadas, possivelmente, em função do emprego de métodos diferentes para o diagnóstico do transtorno, que variam desde a simples avaliação dos sintomas do TDAH até a rigorosa aplicação dos critérios diagnósticos listados nas várias edições do DSM. Além disso, alguns estudos utilizaram versões antigas do DSM (versões II e III), caracterizadas pela inclusão de diferentes critérios diagnósticos para o transtorno. Outro ponto que também justificaria os diferentes resultados encontrados seria a falta de controle dos efeitos de comorbidades psiquiátricas, frequentemente associadas ao TDAH, tais como o transtorno oposicional desafiante, bem como transtornos depressivos e de ansiedade, que podem ser responsáveis pelo aparecimento das alterações do ciclo vigília/sono encontradas nas crianças com TDAH. Por fim, o não controle ou exclusão das crianças que fazem uso de medicação seria outro fator capaz de influenciar os resultados obtidos, já que tratamentos farmacológicos podem afetar os padrões de sono e alerta dos pacientes. Sendo assim, a utilização de sujeitos não-medicados parece mais adequada para avaliar a relação entre os padrões de sono-vigília e o $\mathrm{TDAH}^{(34)}$.

Cortese et al avaliaram todos os artigos que relacionavam TDAH e padrões de sono-vigília em crianças no PubMed entre 1987 (ano da publicação do DSM-III-R) até outubro de 2005. Entre os 46 artigos publicados, apenas 13 (que não possuíam problemas metodológicos que os autores da revisão julgassem capazes de interferir nos resultados) foram 
incluídos em uma meta-análise. Essa meta-análise revelou que nenhum dos cinco estudos que realizaram medidas objetivas dos padrões de sono encontrou diferenças na latência inicial de sono ou na arquitetura do sono de crianças com e sem TDAH. Por esse motivo, os autores sugerem que as alterações no sono encontradas em outros estudos podem ser determinadas por comorbidades ou medicamentos, não sendo causadas, aparentemente, pela presença do transtorno. Por outro lado, o número de movimentos realizados durante o sono foi significantemente maior em crianças com TDAH, quando comparadas aos controles nos três estudos nos quais foi realizada essa avaliação.

No ano de 2009, os mesmos autores publicaram um novo artigo no qual revelaram os resultados de uma nova metaanálise, a qual incluiu os dados utilizados na meta-análise anterior, além dos resultados de outros seis estudos disponíveis no PubMed entre os meses de outubro de 2005 a novembro de 2008 que atendiam aos mesmos critérios de inclusão e exclusão utilizados na publicação de 2006. Essa nova metaanálise revelou que, segundo relatos dos pais, crianças com TDAH apresentam maior resistência a se deitarem, mais dificuldade para iniciar o sono, maior número de despertares noturnos e mais dificuldade para despertar pela manhã do que crianças que não possuem o transtorno. Segundo os autores, a dificuldade para iniciar o sono e a resistência à ida para a cama poderiam ser justificadas pela latência inicial de sono encontrada, maior em crianças com TDAH. Com relação às demais medidas objetivas, houve diferenças significativas entre o grupo de crianças com TDAH e o grupo controle no tempo real de sono, obtido pela actimetria, e eficiência de sono, avaliada pela polissonografia, indicando que crianças com TDAH possuem maior latência inicial de sono, menor duração total de sono e, portanto, menor eficiência de sono. Com relação à arquitetura do sono, diferenças conceituais e metodológicas entre os estudos não permitiram que fossem feitas considerações a respeito, embora o sono de crianças com TDAH pareça ser mais fragmentado e comprometido. Também não foram encontradas diferenças na porcentagem total de cada um dos diferentes estágios de sono, embora a organização de cada ciclo de sono pareça ser diferente entre crianças com e sem TDAH ${ }^{(35)}$.

Entre os estudos incluídos na meta-análise elaborada pelo grupo de Cortese, encontra-se o trabalho de Gruber $e t$ al ${ }^{(36)}$ que, em 2009, publicaram os resultados de 15 crianças com TDAH, livres de medicação e de estimulantes à base de cafeína durante os sete dias que precederam o exame e livres também de comorbidades associadas ao transtorno, que foram submetidas à polissonografia. A diferença deste para outros estudos consistiu na realização do exame na casa do paciente, já que o grupo de pesquisadores dispunha de um polissonígrafo portátil. Dessa forma, pôde-se avaliar o sono das crianças em ambiente familiar, de modo que fatores que podem interferir no sono diário estivessem presentes. Os resultados obtidos com relação aos padrões de sono desses pacientes foram comparados com os resultados obtidos em 23 crianças saudáveis, as quais formaram o grupo controle. O grupo de crianças com TDAH exibiu significativa diminuição na duração de sono, menor duração de sono REM e menor porcentagem de sono REM em relação ao tempo total de sono do que as crianças do grupo controle, não havendo diferenças significativas na duração, latência ou porcentagens dos estágios 1 a 4 de sono entre os grupos. Também não foram notadas associações entre TDAH e transtornos respiratórios durante o sono ou movimentação periódica dos membros. Segundo esses autores, a menor duração de sono REM, encontrada nas crianças com TDAH, poderia ser justificada pela existência de prejuízos no sistema dopaminérgico, que está relacionado tanto a mecanismos de atenção como a mecanismos especificamente associados ao sono $\mathrm{REM}^{(36)}$.

Mais recentemente, Silvestri ${ }^{(37)}$ estudou 55 crianças com TDAH, submetendo-as a videopolissonografia para avaliar possíveis distúrbios de sono. Dos 55 pacientes, 14 crianças apresentaram a síndrome das pernas inquietas (SPI), um distúrbio neurológico caracterizado por uma proeminente necessidade de movimentação das pernas, com piora dos sintomas quando o indivíduo se encontra em repouso ${ }^{(38)}$. Observando-se o total de sujeitos diagnosticados com TDAH e síndrome das pernas inquietas e os portadores do TDAH que não apresentavam a síndrome, os autores notaram correlação positiva entre a presença do transtorno associado à SPI e o comportamento hiperativo, sendo que sujeitos diagnosticados com TDAH, subtipo hiperativo ou combinado apresentavam maior gravidade da síndrome do que aqueles diagnosticados com TDAH, subtipo desatento. Esses resultados são similares aos encontrados por Chervin et $a^{(39)}$, em estudo que avaliou as associações entre hiperatividade, desatenção, SPI e movimentação periódica das pernas em crianças.

São muitas as hipóteses que justificam a associação entre sintomas de desatenção e agitação motora e de membros durante o sono, sendo que o incômodo causado pela síndrome das pernas inquietas poderia gerar dificuldades para iniciar o sono. Além disso, a agitação desencadeada pela presença da síndrome das pernas inquietas poderia gerar 
pequenos despertares, os quais prejudicariam a arquitetura do sono ${ }^{(40)}$. Segundo Owens, distúrbios de sono na infância podem se apresentar por meio de sonolência diurna, tal como em adultos, ou por meio da manifestação de sintomas neurocomportamentais, muitos dos quais se sobrepõem aos sintomas do $\operatorname{TDAH}^{(9)}$. Bioquimicamente, a associação entre síndrome das pernas inquietas e TDAH poderia ser explicada pela presença de alterações dopaminérgicas, que, segundo alguns estudos, ocorrem em ambos os casos. Além disso, sugere-se que a deficiência de ferro possa também se relacionada aos dois problemas, pois o íon ferro atua como cofator da enzima tirosina hidroxilase, envolvida na síntese de dopamina ${ }^{(40)}$. De fato, Cortese et al, investigando das possíveis associações entre os níveis de ferro e os padrões de sono em crianças com TDAH, observaram que crianças cujos níveis séricos de ferro mantinham-se abaixo de $45 \mu \mathrm{g} / \mathrm{L}$ obtinham, a partir das respostas dadas pelos pais, maiores pontuações na Escala de Distúrbios de Sono para Crianças, sendo que, entre os itens avaliados, encontra-se a movimentação anormal durante o sono ${ }^{(35)}$. Entretanto, segundo os autores, pesquisas envolvendo medidas objetivas dos padrões de sono são necessárias para constatar essa possível relação.

\section{Referências bibliográficas}

1. Stefanatos GA, Baron IS. Attention-deficit/hyperactivity disorder: a neuropsychological perspective towards DSM-V. Neuropsychol Rev 2007; 17:5-38.

2. Pereira HS, Araújo AP, Mattos P. Attention-deficit hyperactivity disorder: aspects related to motor activity comorbidity. Rev Bras Saude Mater Infant 2005:5:391-402.

3. Díaz-Heijtz R. Biochemical bases and research in attention-deficit hyperactivity disorder. Rev Neurol. 2002;34 (Suppl 1):S78-81.

4. Kirov R, Kinkelbur J, Heipke S, Kostanecka-Endress T, Westhoff M, Cohrs $S$ et al. Is there a specific polysomnographic sleep pattern in children with attention deficit/hyperactivity disorder? J Sleep Res 2004;13:87-93.

5. Huang YS, Chen NH, Li HY, Wu YY, Chao CC, Guilleminault C. Sleep disorders in Taiwanese children with attention deficit/hyperactivity disorder. J Sleep Res 2004;13:269-77.

6. Owens J, Sangal RB, Sutton VK, Bakken R, Allen AJ, Kelsey D. Subjective and objective measures of sleep in children with attention-deficit/hyperactivity disorder. Sleep Med 2009;10:446-56.

7. Sung V, Hiscock H, Sciberras E, Efron D. Sleep problems in children with attention-deficit/hyperactivity disorder: prevalence and the effect on the child and family. Arch Pediatr Adolesc Med 2008;162:336-42.

8. American Academy of Pediatrics. Clinical practice guideline: diagnosis and evaluation of the child with attention-deficit/hyperactivity disorder. Pediatrics 2000;105:1158-70.

9. Owens JA. A clinical overview of sleep and attention-deficit/hyperactivity disorder in children and adolescents. J Can Acad Child Adolesc Psychiatry 2009;18:92-102.

\section{Conclusão}

A partir dos dados apresentados anteriormente, constata-se que a associação entre padrões de sono e atividade motora ainda necessita ser investigada, pois alguns achados corroboram a ideia de que, pelo menos em um subgrupo de pacientes, o TDAH pode ter duração de 24 horas, ou seja, os sintomas de hiperatividade poderiam permanecer durante o sono, com maior movimentação nesse período. Outros, entretanto, sugerem que a perturbação do sono causada pelos movimentos executados pela criança adormecida poderia contribuir para a sintomatologia diurna do TDAH. Segundo a teoria proposta por Weinberg e Harper, a manifestação de sintomas de hiperatividade seria uma forma de contornar a sonolência excessiva que as crianças com TDAH apresentam, dados os diversos problemas de sono que possuem ${ }^{(35,40)}$.

Uma análise mais detalhada das relações existentes entre essas variáveis pode fornecer subsídios para compreender o papel do sono em alterações da atividade motora e, consequentemente, elucidar a participação de alterações do ciclo vigília/ sono na etiologia do TDAH, o que poderia contribuir para o desenvolvimento de novas formas de tratamento e melhoria da qualidade de vida dos pacientes e de seus familiares.

10. Rohde LA, Barbosa G, Tramontina S, Polanczyk G. Transtorno de déficit de atenção/hiperatividade. Rev Bras Psiquiatr 2000;22 (Supl 2):7-11.

11. Cormier E. Attention deficit/hyperactivity disorder: a review and update. $J$ Pediatr Nurs 2008;23:345-57.

12. Fontana Rda S, Vasconcelos MM, Jr Werner J Jr, Góes FV, Liberal EF. ADHD prevalence in four Brazilian public schools. Arq Neuropsiquiatr 2007;65:134-7.

13. Singh I. Beyond polemics: science and ethics of ADHD. Nat Rev Neurosci 2008;9:957-64.

14. Biederman J. Attention-deficit/hyperactivity disorder: a selective overview. Biol Psychiatry 2005;57:1215-20.

15. Sangal RB, Owens J, Allen AJ, Sutton V, Schuh K, Kelsey D. Effects of atomoxetine and methylphenidate on sleep in children with ADHD. Sleep 2006;29:1573-85.

16. Tomás Vila M, Miralles Torres A, Beseler Soto B, Revert Gomar M, Sala Langa MJ, Uribelarrea Sierra Al. Relación entre el trastorno por déficit de atención e hiperactividad y los trastornos del sueño. Resultados de un estudio epidemiológico en la población escolar de la ciudad de Gandía. An Pediatr (Barc) 2008;69:251-7.

17. Greene R, Siegel J. Sleep: a functional enigma. Neuromolecular Med 2004;5:59-68.

18. Walker MP, Stickgold R. Sleep, memory, and plasticity. Annu Rev Psychol 2006; 57:139-66.

19. Rial RV, Nicolau MC, Gamundí A, Akaârir M, Aparicio S, Garau C et al. The trivial function of sleep. Sleep Med Rev 2007;11:311-25.

20. Scharf MT, Naidoo N, Zimmerman JE, PackAI. The energy hypothesis of sleep 
revisited. Prog Neurobiol 2008;86:264-80.

21. Knutson KL, Spiegel K, Penev P, Van Cauter E. The metabolic consequences of sleep deprivation. Sleep Med Rev 2007;11:163-78.

22. Graves LA, Heller EA, Pack AI, Abel T. Sleep deprivation selectively impairs memory consolidation for contextual fear conditioning. Learn Mem 2003;10:168-76.

23. Banks S, Dinges DF. Behavioral and physiological consequences of sleep restriction. J Clin Sleep Med 2007;3:519-28.

24. Sadeh A, Gruber R, Raviv A. The effects of sleep restriction and extension on school-age children: what a difference an hour makes. Child Dev 2003;74:444-55.

25. Hill CM, Hogan AM, Karmiloff-Smith A. To sleep, perchance to enrich learning? Arch Dis Child 2007;92:637-43.

26. Nixon GM, Thompson JM, Han DY, Becroft DM, Clark PM, Robinson E et al. Short sleep duration in middle childhood: risk factors and consequences. Sleep 2008;31:71-8.

27. Thunström M. Severe sleep problems in infancy associated with subsequent development of attention-deficit/hyperactivity disorder at 5.5 years of age. Acta Paediatr 2002;91:584-92.

28. Touchette E, Petit D, Séguin JR, Boivin M, Tremblay RE, Montplaisir JY. Associations between sleep duration patterns and behavioral/cognitive functioning at school entry. Sleep 2007;30:1213-9.

29. Lam LT, Yang L. Duration of sleep and ADHD tendency among adolescents in China. J Atten Disord 2008;11:437-44.

30. Biederman J, Spencer T. Attention-deficit/hyperactivity disorder (ADHD) as a noradrenergic disorder. Biol Psychiatry 1999;46:1234-42.

31. Lee JS, Kim BN, Kang E, Lee DS, Kim YK, Chung JK et al. Regional cerebral blood flow in children with attention deficit hyperactivity disorder: comparison before and after methylphenidate treatment. Hum Brain Mapp 2005;24:157-64
32. O'brien LM, Holbrook CR, Mervis CB, Klaus CJ, Bruner JL, Raffield TJ et al. Sleep and neurobehavioral characteristics of 5- to-7-year-old children with parentally reported symptoms of attention-deficit/hyperactivity disorder. Pediatrics 2003;111:554-63.

33. Konofal E, Lecendreux M, Bouvard MP, Mouren-Simeoni MC. High levels of nocturnal activity in children with attention-deficit hyperactivity disorder: a video analysis. Psychiatry Clin Neurosci 2001;55:97-103.

34. Cortese S, Konofal E, Yateman N, Mouren MC, Lecendreux M. Sleep and alertness in children with attention-deficit/hyperactivity disorder: a systematic review of the literature. Sleep 2006;29:504-11.

35. Cortese S, Faraone SV, Konofal E, Lecendreux M. Sleep in children with attention-deficit/hyperactivity disorder: meta-analysis of subjective and objective studies. J Am Acad Child Adolesc Psychiatry 2009;48:894-908.

36. Gruber R, Xi T, Frenette S, Robert M, Vannasinh P, Carrier J. Sleep disturbances in prepubertal children with attention deficit hyperactivity disorder: a home polysomnography study. Sleep 2009;32:343-50.

37. Silvestri R, Gagliano A, Aricò I, Calarese T, Cedro C, Bruni O et al. Sleep disorders in children with Attention-Deficit/Hyperactivity Disorder (ADHD) recorded overnight by video-polysomnography. Sleep Med 2009;10:1132-8.

38. Picchietti MA, Picchietti DL. Restless legs syndrome and periodic limb movement disorder in children and adolescents. Semin Pediatr Neurol 2008;15:91-9.

39. Chervin RD, Archbold KH, Dillon JE, Pituch KJ, Panahi P, Dahl RE et al. Associations between symptoms of inattention, hyperactivity, restless legs, and periodic leg movements. Sleep 2002;25:213-8.

40. Walters AS, Silvestri R, Zucconi M, Chandrashekariah R, Konofal E. Review of the possible relationship and hypothetical links between attention deficit hyperactivity disorder (ADHD) and the simple sleep related movement disorders, parasomnias, hypersomnias and circadian rhythm disorders. J Clin Sleep Med 2008;4:591-600. 